\title{
Missense mutation in the ATPase, aminophospholipid transporter protein ATP8A2 is associated with cerebellar atrophy and quadrupedal locomotion
}

\author{
Onur Emre Onat ${ }^{1,10}$, Suleyman Gulsuner ${ }^{1,10}$, Kaya Bilguvar ${ }^{2,3,4}$, Ayse Nazli Basak ${ }^{5}$, Haluk Topaloglu ${ }^{6}$, \\ Meliha $\operatorname{Tan}^{7}$, Uner $\operatorname{Tan}^{8}$, Murat Gunel ${ }^{2,3,4}$ and Tayfun Ozcelik ${ }^{\star, 1,9}$
}

Cerebellar ataxia, mental retardation and dysequilibrium syndrome is a rare and heterogeneous condition. We investigated a consanguineous family from Turkey with four affected individuals exhibiting the condition. Homozygosity mapping revealed that several shared homozygous regions, including chromosome 13q12. Targeted next-generation sequencing of an affected individual followed by segregation analysis, population screening and prediction approaches revealed a novel missense variant, p.I376M, in ATP8A2. The mutation lies in a highly conserved C-terminal transmembrane region of E1 E2 ATPase domain. The ATP8A2 gene is mainly expressed in brain and development, in particular cerebellum. Interestingly, an unrelated individual has been identified, in whom mental retardation and severe hypotonia is associated with a de novo $t(10 ; 13)$ balanced translocation resulting with the disruption of ATP8A2. These findings suggest that ATP8A2 is involved in the development of the cerebrocerebellar structures required for posture and gait in humans.

European Journal of Human Genetics (2013) 21, 281-285; doi:10.1038/ejhg.2012.170; published online 15 August 2012

Keywords: ATP8A2; cerebellar hypoplasia; targeted next-generation sequencing; quadrupedal locomotion; CAMRQ

\section{INTRODUCTION}

Cerebellar ataxia, mental retardation and dysequilibrium syndrome (CAMRQ) is a rare and genetically heterogeneous autosomal recessive disorder characterized by mental retardation, cerebellar ataxia and dysarthric speech with or without quadrupedal gait. ${ }^{1-8}$ Multiple consanguineous families have been reported with autosomal recessive inheritance of the condition. The first locus was mapped to a $7.1-\mathrm{Mb}$ region on chromosome $17 \mathrm{p} 13$ and a missense mutation was reported on WDR81 (WD repeat domain 81; CAMRQ2; MIM: 610185; also referred to as Uner Tan syndrome). ${ }^{1,2,7}$ Linkage mapping followed by candidate gene sequencing also led to the identification of mutations in very low-density lipoprotein receptor (CAMRQ1; MIM: $224050)^{3-5}$ and carbonic anhydrase VIII (CAMRQ3; MIM: 613227). ${ }^{6}$

In another consanguineous family (Family C) ${ }^{3,9}$ from Turkey, the involvement of VLDLR, WDR81 and CA8 genes were excluded, and four shared-homozygous regions on chromosomes 13, 19 and 20 were uncovered by homozygosity mapping. To identify the culprit gene, we utilized targeted next-generation sequencing of all homozygous regions and evaluated all co-segregated variants using functional and structural predictions and population screening. We report herein that a recessive missense mutation in ATP8A2, encoding ATPase, aminophospholipid transporter, class $\mathrm{I}$, type $8 \mathrm{~A}$, member 2 , is associated with the phenotype in Family C. In an independent study, a de novo $\mathrm{t}(10 ; 13)$ balanced translocation disrupting the coding sequence of ATP8A2 on 13q12 was observed in a patient with severe mental retardation and major hypotonia, raising the possibility that haploinsufficiency of this gene could be implicated in neurodevelopmental phenotypes. ${ }^{10}$ On the basis of these observations, we suggest that ATP8A2 could be critically important in the development of the nervous system.

\section{SUBJECTS AND METHODS}

Patients

The consanguineous family analyzed in this study has four members affected by mental retardation, mild cerebellar and cerebral atrophy and truncal ataxia (Figure 1). The index case was a 27-year-old man exhibiting total inability to walk (05-993). Briefly, patients share the following clinical features: truncal ataxia with/without quadrupedal gait, mental retardation and dysarthric speech. MRI results revealed mild atrophy of cerebral cortex, corpus callosum and inferior cerebellum. Clinical description of Family $\mathrm{C}$ was published elsewhere., ${ }^{3,9}$ The only affected female in the family could not be included in the study, as her parents did not give consent for DNA analysis. Case 05-993 recently died secondary to a respiratory infection. The study was approved by the institutional review boards at the Baskent and Cukurova Universities (decision KA07/47, 02.04.2007 and 21/3, 08.11.2005, respectively). Written informed consent was obtained from all participants or their parents before the study.

${ }^{1}$ Department of Molecular Biology and Genetics, Faculty of Science, Bilkent University, Ankara, Turkey; ${ }^{2}$ Department of Neurosurgery, Yale University School of Medicine, New Haven, CT, USA; ${ }^{3}$ Department of Neurobiology, Yale University School of Medicine, New Haven, CT, USA; ${ }^{4}$ Department of Genetics, Center for Human Genetics and Genomics and Program on Neurogenetics, Yale University School of Medicine, New Haven, CT, USA; ${ }^{5}$ Department of Molecular Biology and Genetics, NDAL Laboratory, School of Arts and Sciences, Bogazici University, Istanbul, Turkey; ${ }^{6}$ Department of Pediatric Neurology, Ihsan Dogramaci Children's Hospital, Hacettepe University Faculty of Medicine, Ankara, Turkey; ${ }^{7}$ Department of Neurology, Baskent University Faculty of Medicine, Ankara, Turkey; ${ }^{8}$ Department of Physiology, Cukurova University Faculty of Medicine, Adana, Turkey; ${ }^{9}$ Institute of Materials Science and Nanotechnology (UNAM), Bilkent University, Ankara, Turkey

*Correspondence: Dr T Ozcelik, Department of Molecular Biology and Genetics, Faculty of Medicine, Bilkent University, Ankara 06800 , Turkey. Tel: +90 312 290 2139;

Fax: +90 312266 5097; E-mail: tozcelik@bilkent.edu.tr

10The first two authors are regarded as joint first authors.

Received 9 March 2012; revised 3 July 2012; accepted 6 July 2012; published online 15 August 2012 


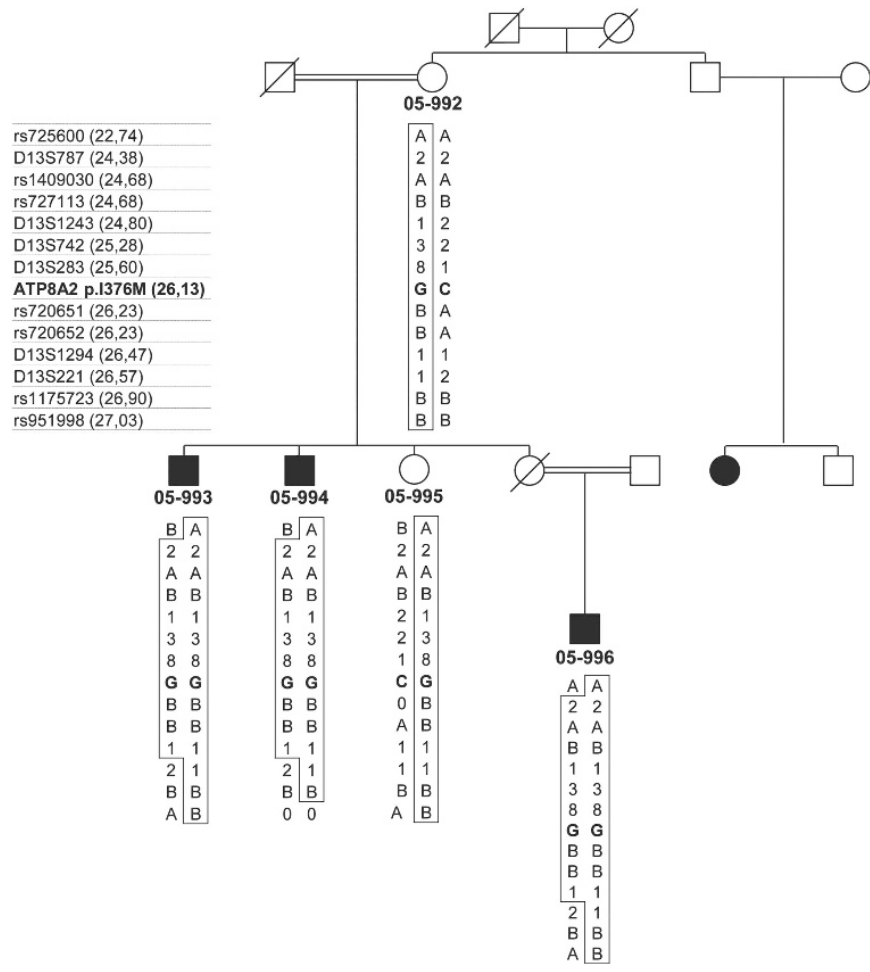

Figure 1 Pedigree of Family $\mathrm{C}$ with haplotype structure of the disease interval on chromosome 13q12. Haplotype segregating with the disease is boxed. ATP8A2 c. $1128 \mathrm{C}>\mathrm{G}$ mutation is bold. Please note that the DNA of one affected individual is not available for the study.

\section{Homozygosity mapping analysis}

Participants' DNA from peripheral blood samples were genotyped using $10 \mathrm{~K}$ Affymetrix SNP chips. Experiments were performed according to the manufacturer's instructions (Affymetrix, Santa Clara, CA, USA). DNA of two affected individuals (05-994 and 05-996) was genotyped using Illumina Human610-Quad BeadChip according to manufacturer's recommendations (Illumina, Inc., San Diego, CA, USA). The image data were normalized and the genotypes were called using data analysis software (Bead Studio, Illumina). Homozygosity mapping analysis was performed using HomozygosityMapper software. ${ }^{11}$ Homozygosity was ruled out for the previously reported loci. Markers D13S787, D13S1243, D13S742, D13S283, D13S1294 and D13S221 were used to test homozygosity for the most likely candidate locus, chromosome 13q12. Haplotype analysis was carried out by hand.

\section{Mutation analysis}

A total of 16711445 base long unique probes were designed to target homozygous regions (Supplementary Table 1) using a custom-designed Nimblegen Human Sequence Capture HD2 microarray (Roche NimbleGen, Madison, WI, USA). DNA sample from an affected individual (05-996) was captured using $3 \mu \mathrm{g}$ input DNA. Captured DNA sample was sequenced with Illumina Genome Analyzer IIx. Illumina sequence data were mapped to reference genome (hg18) using $\mathrm{Maq}^{12}$ and single-nucleotide variants were determined with Samtools. ${ }^{13}$ To determine indels, data were mapped with BWA $^{14}$ and analyzed with Samtools. Variant coordinates were converted to hg19 before publication by liftOver tool (http://genome.ucsc.edu/cgi-bin/ hgLiftOver). Coverage calculations of coding regions were done with mpileup module of Samtools ${ }^{13}$ and intersectBED command of BEDTools. ${ }^{15}$ Novel variants were determined based on SNPs reported in dbSNP database and further analyzed in 1000 genome data sets (http://www.1000genomes.org), NHLBI Exome Sequencing Project (http://evs.gs.washington.edu/EVS/, data release ESP5400) and exome sequencing data of 2400 individuals with nonneurological disorders generated at Yale University. Common variants were excluded if minor allele frequency was lower than $0.1 \%$. Novel variants were confirmed by Sanger sequencing. Segregation analysis of the variants in the pedigree and its presence in healthy population were carried out using allelespecific PCR analysis (Supplementary Table 2). Racial distribution of control group was $100 \%$ Caucasian, including 22\% from southeastern Turkey.

\section{Bioinformatics analysis}

DNA and protein sequences were obtained from ENSEMBL database. ${ }^{16}$ SIFT, ${ }^{17}$ PolyPhen $2^{18}$ and MutationTaster ${ }^{19}$ tools were used to predict causative variants. Genomic evolutionary rate profiling (GERP) and phylogenetic $P$-value (phyloP) conservation scores for each variant were extracted seperately from the UCSC Genome Browser allHg19RS_BW track ${ }^{20}$ and phyloP46wayall track, ${ }^{21}$ respectively. Functional and transmembrane domains of the ATP8A2 protein were predicted using Pfam database ${ }^{22}$ and TmPred prediction tool (www.ch.embnet.org/software/TMPRED_form.html), respectively. Homology searches were performed with CLCMain Workbench (CLC Bio, Aarhus, Denmark) using appropriate modules. CLCMain Workbench also generates phylogenetic tree using UPGMA algorithm that is evaluated by bootstrap analysis. Possible effects of the variant on protein secondary structure were predicted using PSIPRED server. ${ }^{23}$ Published microarray data sets of E9.5, E11.5 and E13.5 mouse brain tissue $\left(\right.$ GSE8091) ${ }^{24}$ were obtained from the GEO database (http:// www.ncbi.nlm.nih.gov/projects/geo/query/acc.cgi) and analyzed with GeneSpring GX V11.1 software (Agilent Technologies, Santa Clara, CA, USA). Differentially expressed genes within day groups were filtered (oneway ANOVA test Bonferroni-corrected $P<0.001)$ and genes that correlated with Atp8a2 $(R=0.95-1.0)$ were functionally annotated using DAVID tools. ${ }^{25}$ Primers used in this study were designed with Primer $3^{26}$ software and are listed in Supplementary Table 2.

\section{Quantitative real-time RT-PCR}

First-strand cDNAs were prepared from human RNA samples (Clontech, Mountain View, CA, USA: 636567 (corpus callosum); Agilent: 540007 (cerebellum), 540117 (frontal cortex), 540137 (occipital cortex), 540157 (fetal brain), 540053 (brain stem), 540005 (total brain), 540143 (parietal cortex), 540135 (striatum)) using RevertAid First Strand cDNA Synthesis kit with random hexamer primers (Fermentas, now Thermo Fisher Scientific, Waltham, MA, USA; K1622) after DNaseI (Fermentas EN0521) digestion. Real-time RTPCR was performed using IQ SYBR Green Supermix according to standard protocols (BioRad, Hercules, CA, USA; 170-8882). $C_{\mathrm{t}}$ values were normalized to $G A P D H$ as an internal control. The data were analyzed using the Pfaffl method. ${ }^{27}$

\section{RESULTS}

We identified four common homozygous regions in two affected individuals (05-994 and 05-996) using Ilumina Human610-Quad BeadChip. Targeted next-generation sequencing of all homozygous regions (Supplementary Figure 1 and Supplementary Table 1) was carried out using DNA of one affected individual (05-996). This region was enriched 629 -fold in the capture experiment. In total, 48.62 million single-end 75 bp reads were obtained and $29.2 \%$ of the reads mapped to the targeted regions. This in turn provided a mean coverage depth of 62.96-fold across the targeted homozygosity intervals with $97.41 \%$ of the targeted bases being covered by at least four reads (Supplementary Table 3). Next, the constitutive exons in the homozygous intervals were analyzed and $99.51 \%$ of the protein coding regions was found to be covered by at least four reads. When the genes encoding for the constitutive exons in the low- or zerocoverage regions were analyzed, they either do not have cerebellar expression or do not display a phenotype compatible with cerebellar involvement in mouse knockouts (Supplementary Table 4). On the basis of these results, we find it highly unlikely that a causative mutation is missed. 
Table 1 Novel coding variants identified by targeted next-generation sequencing of 05-996

\begin{tabular}{|c|c|c|c|c|c|c|c|c|c|c|}
\hline Gene & Position (hg19) & Ref & Var & Effect & GERP (score) & PhyloP (score) & SIFT (score) & Polyphen2 (score) & M. Taster (P-value) & Segregation \\
\hline АТР8А2 & chr13:26,128,001 & C & $\mathrm{G}$ & $1376 \mathrm{M}$ & 2.18 & 1.091 & D. $(0.02)$ & P.D. (1.00) & D.C. (0.995) & Yes \\
\hline APBA3 & chr19:3,759,974 & $\mathrm{C}$ & $\mathrm{T}$ & А97Т & -4.11 & -0.308 & T. $(0.16)$ & B. $(0.14)$ & P. (0.999) & Yes \\
\hline MUC16 & chr19:9,068,391 & G & $A$ & A6352V & -1.45 & -0.803 & n.a. & n.a. & P. (0.999) & No \\
\hline MUC16 & chr19:9,068,577 & $\mathrm{G}$ & $A$ & T6290I & 2.35 & 2.273 & n.a. & n.a. & P. (0.999) & No \\
\hline ZNF823 & chr19:11,833,601 & $A$ & $\mathrm{G}$ & C250R & 0.632 & 1.532 & D. $(0.00)$ & P.D. (1.00) & P. $(0.994)$ & No \\
\hline SERINC3 & chr20:43,141,490 & $A$ & $\mathrm{G}$ & M116T & 3.98 & 2.524 & T. $(0.34)$ & B. $(0.13)$ & D.C. (0.999) & No \\
\hline PCP2 & chr19:7,698,326 & СТC & - & E6del & n.a. & 0.168 & n.a. & n.a. & P. $(0.717)$ & Yes \\
\hline
\end{tabular}

Abbreviations: Ref, reference allele; Var, variant allele; M.Taster, Mutation Taster, D., damaging; T., tolerated; P.D., probably damaging; B., benign; n.a., not available; D.C., disease causing; P., polymorphism.

A total of 14103 homozygous variants (13394 single-nucleotide variants and 709 indels) were detected by next-generation sequencing. Of these, 13528 variants were reported by dbSNP132. Remaining 575 novel variants were classified by genomic context: protein altering or flanking splice junctions $(n=11)$, coding synonymous $(n=4)$, $5^{\prime}$-UTR $(n=44), 3^{\prime}$-UTR $(n=30)$, intronic $(n=224)$ and intergenic $(n=262)$. Of the 11 protein-altering variants, four were excluded based on the comparison for novelty with 1000 genomes data, NHLBI Exome Sequencing Project and the exome sequence data of 2400 individuals with non-neurological diseases. The remaining seven variants in the coding regions of homozygous blocks were verified by Sanger sequencing and four of them were excluded by segregation analysis (Supplementary Figures 2-3). Two missense variants (ATP8A2 p.I376M and APBA3 p.A97T) and a 3-bp in-frame deletion (PCP2 p.E6del) were consistent with the recessive inheritance of the disease allele in Family C (Table 1, Figure 1 and Supplementary Figure 2).

APBA3 p.A97T variant was excluded based on the conservation considerations and prediction analyses. Four of 20 species sequenced have threonine (T) at the orthologous site (Supplementary Figure 4), suggesting that this variant would be a polymorphism and not damaging to humans. A negative GERP score $(-4.11)$ for the mutated nucleotide suggests that this site is probably evolving neutrally. ${ }^{20}$ PhyloP score of the variant $(-0.308)$ suggests a faster evolution than expected for this site. ${ }^{21}$ Furthermore, the variant was predicted as 'tolerated' by SIFT $^{17}$ (SIFT score, 0.16), 'benign' by PolyPhen $2^{18}$ (PSIC score difference, 0.0) and 'polymorphism' by MutationTaster ${ }^{19}$ ( $P$-value, 0.999) (Table 1).

PCP2 p.E6del was excluded based on population screening. In 360 healthy chromosomes, four heterozygous individuals were identified (Supplementary Figure 5), yielding an expected homozygote frequency of approximately 1 in 8000 . The region containing the mutation is not conserved among species, and the deletion was predicted as 'polymorphism' by MutationTaster ${ }^{19}$ ( $P$-value, 0.717; Table 1 and Supplementary Figure 6).

The remaining variant at chr13:26128001 (hg19; c.1128 C>G) is located in exon 12 of ATP8A2 (ENSG00000132932, ENST00000381655) and results in an isoleucine (I) to methionine (M) substitution at residue 376 . The mutation co-segregated with the disease in Family C (Figure 1) lies in the C-terminal-predicted transmembrane site of the E1 E2 ATPase domain (Figure 2a) and is highly conserved across species (Figure $2 \mathrm{~b}$ and Supplementary Figure 7). Screening of 1210 control chromosomes, including 300 individuals from the same geographic region as Family C, excluded presence of the variant in this control population. SIFT, ${ }^{17}$ PolyPhen $2^{18}$ and MutationTaster ${ }^{19}$ tools predicted the ATP8A2 p.I376M as a causative mutation (scores: 0.0, 1.0 and 0.955, respectively). Consequences of the amino acid change in protein structure were evaluated by comparing the predicted secondary structures of wildtype and mutant protein sequences. The wild-type protein is predicted to contain $27 \beta$-strands and $32 \alpha$-helices. I376 residue is located at the $\mathrm{N}$ terminus of the 11th $\alpha$-helix. The mutation enlarges the 11th and 12th $\alpha$-helices and creates an additional $\alpha$-helix at residue 401 (Figure 2c).

The status of ATP8A2 was evaluated in a cohort of 750 patients with structural cortical malformations or degenerative neurological disorders, and the underlying genetic cause is still unknown. Wholegenome genotyping data generated by Illumina Human 370 Duo or $610 \mathrm{~K}$ Quad BeadChips is available for this cohort. None of the patients were found to harbor a homozygous interval $(\geq 2.5 \mathrm{cM})$ surrounding the ATP8A2 locus. Exome sequencing of the same group did not reveal any mutations, including compound heterozygous substitutions, in ATP8A2.

The transmembrane protein, ATP8A2, consists of four proteincoding isoforms. The longest isoform (ENST00000381655) contains 37 exons and encodes a $112 \mathrm{kDa}$ protein. The protein is highly expressed in newborn and embryonic tissues, with strongest expression in mouse heart, brain and testis. ${ }^{10,28}$ RT-PCR analysis revealed similar expression in different regions of the human brain. ${ }^{10}$ To evaluate the possible involvement of ATP8A2 in motor functions, we examined its expression profile in different human brain regions by quantitative real-time RT-PCR. Human ATP8A2 is expressed in all brain regions with the highest level of expression in cerebellum (Figure 3). ATP8A2 expression in the patients cannot be evaluated, as the gene is not expressed in lymphocytes.

To further investigate the role of ATP8A2 in brain development, we examined the expression profiles of early embryonic mouse brain $\left(\right.$ GSE8091) ${ }^{24}$ and identified genes with significantly correlated expression profiles $(R>0.95, n=218)$ with that of ATP8A2. Functional clustering analysis suggested that positively correlated genes were enriched for those involved in neuron differentiation, cell, and neuron projection morphogenesis and axonogenesis (Bonferroni-corrected $P$-values: $2.1 \mathrm{E}-3,2.7 \mathrm{E}-3,4.5 \mathrm{E}-3$ and $1.5 \mathrm{E}-2$ respectively). ATP8A2 is co-expressed with doublecortin responsible for lissencephaly and WDR81 associated with CAMRQ2, ${ }^{7}$ suggesting that these genes could represent similar developmental pathways.

\section{DISCUSSION}

CAMRQ is a rare genetically heterogeneous cerebellar ataxia with mental retardation and dysarthric speech, with or without quadrupedal gait. Since the first mapping of the gene locus on chromosome 17p13, two additional loci on chromosomes 9p24 and 8q12 have been reported, and causative mutations have been identified in VLDLR, CA8 and WDR81. 2,3,6,7 Here we present the identification of a fourth gene locus in a consanguineous family of two affected 


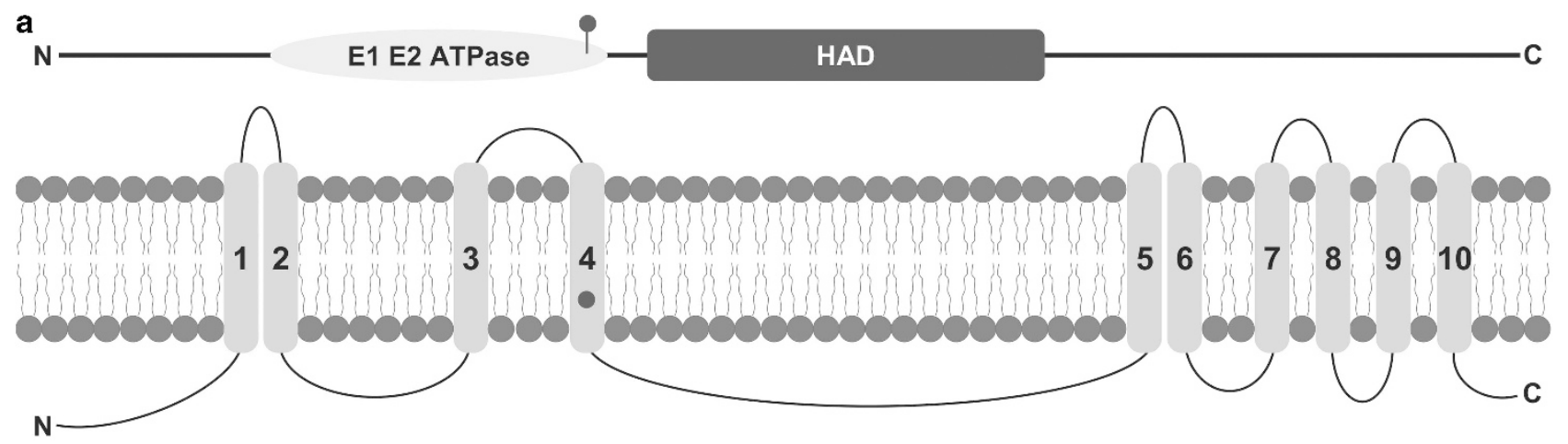

b

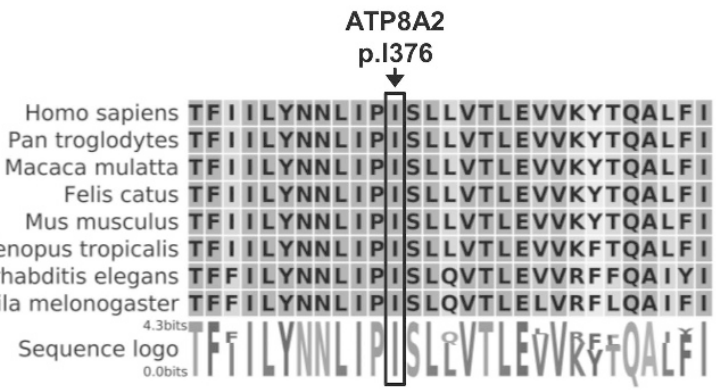

c

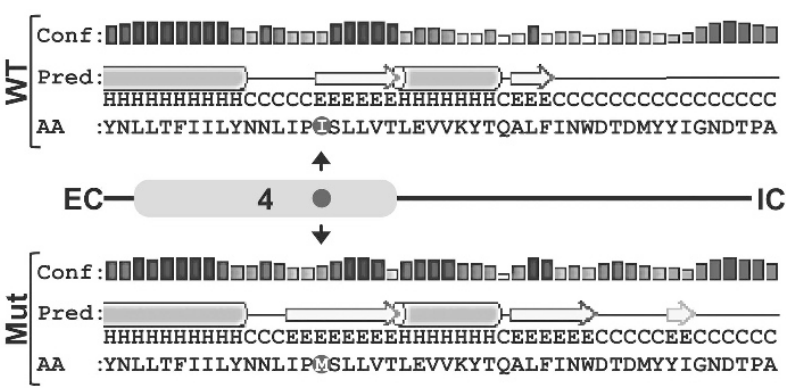

Figure 2 Graphical representation of the predicted functional and structural elements of ATP8A2 protein. (a) ATP8A2 is composed of an E1 E2 ATPase domain and a haloacid dehalogenase-like hydrolase (HAD) domain. Ten transmembrane domains were predicted by TMPRED. The mutation lies in the transmembrane region of C-terminal end of E1 E2 ATPase domain (dot). (b) Multiple amino acid sequence alignments show the sequence homology of ATP8A2 protein in vertebrates. 1376 residue is indicated with a box. (c) Graphical representation of secondary structural elements as predicted by PSIPRED. The predicted elements (Pred) are indicated above the amino acid (AA) sequences (straight lines: coils; cylinders: helices; arrows: strands). The mutation is predicted to alter the secondary structure of the protein. Transmembrane region is represented within the Pred graphs of wild-type (WT) and mutant (Mut) proteins. EC, extracellular; IC, intracellular.

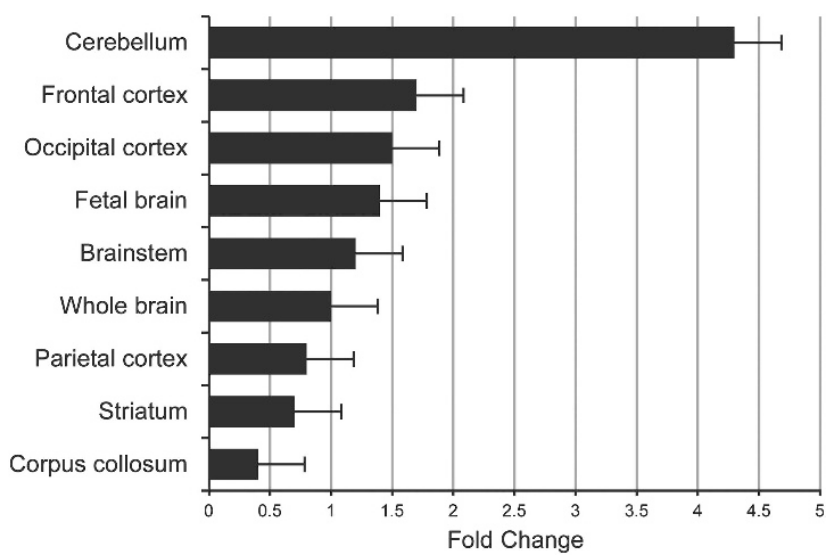

Figure 3 Expression pattern of ATP8A2 in nine different regions of human brain. Real-time RT-PCR analysis showed that ATP8A2 is expressed in all regions of the brain with the highest levels in the cerebellum.

siblings and an affected nephew. Using whole-genome homozygosity mapping followed by targeted next-generation sequencing, several missense variants were observed. Filtering the variants by co-segregation analysis, population screening, protein conservation and disease gene prediction approaches revealed a novel missense variant in ATP8A2 (c.1128 C> G; p.I376M) that segregates with the phenotype. The mutation is located inside a transmembrane domain and is predicted to change secondary structure of the protein.
ATP8A2 belongs to the $\mathrm{P}_{4}$-ATPases subfamily of P-type ATPases, which are involved in the transport of aminophospholipids. Biochemical studies have shown that $\mathrm{P}_{4}$-ATPases determine the curvature of the phospholipid bilayer by flipping aminophospholipids from the exoplasmic to the cytoplasmic leaflet. ${ }^{29,30}$ ATPases have been implicated in human diseases such as ATP10C in Angelman syndrome, ${ }^{31}$ ATP8B1 in hearing loss ${ }^{32}$ and hereditary cholestasis, ${ }^{33}$ and $A T P 8 A 2$ in a severe neurological phenotype. ${ }^{10}$

ATP8A2 is involved in the transport of aminophospholipids toward the cytoplasmic leaflet in brain cells, retinal photoreceptors and testis. ${ }^{34}$ In humans, ATP8A2 is mainly expressed in brain tissues, with highest levels in cerebellum, as well as in retina and testis. ${ }^{10}$ Cerebellum is a crucial regulatory organ for motor coordination and this expression pattern is consistent with CAMRQ. The fact that CAMRQ-associated genes have retinal expression ${ }^{34,35}$ raises the possibility that eye abnormalities may be an additional clinical feature of the phenotype. Strabismus has been observed in almost all affected individuals in all the families reported thus far. ${ }^{1-8}$ In addition, homozygous WDR81 mutation carriers display downbeat nystagmus, temporal disk pallor and macular atrophy. ${ }^{36}$ However, retinopathy is not a feature of WDR81-, VLDLR- and CA8-associated CAMRQ. ${ }^{6,36}$ With respect to ATP8A2, further information is not available, as Family $\mathrm{C}$ declined neuro-ophthalmological investigations.

Documentation of a de-novo-balanced translocation leading to ATP8A2 haploinsufficiency ${ }^{10}$ brings into attention the clinical findings of carriers in Family C. Whereas 05-992 and 05-995 did not show neurological abnormalities, the $\mathrm{t}(10 ; 13)$ de-novo-balanced translocation carrier presented with a severe neurological phenotype 
that partially overlaps with the phenotype of the affected members of Family C. The possibility of a chimeric protein was ruled out, leaving haploinsufficiency of ATP8A2 as the most likely explanation for the phenotype. This suggests that ATP8A2 mutations represent yet another example of clinical heterogeneity in the context of genomic understanding of complex traits in humans and demonstrates fundamental features of genomic analysis of human traits such as variable expression, allelic heterogeneity and genotype-phenotype correlations. Other examples include $C R Y B B 1$ in congenital cataract, ${ }^{37}$ COLL11A2 in Zweymuller Weissenbacher syndrome ${ }^{38}$ and MYBPC1 in arthrogryposis. ${ }^{39}$

These findings suggest that ATP8A2 could be critical for the developmental processes of central nervous system, and alterations of this gene may lead to severe neurological phenotypes.

\section{CONFLICT OF INTEREST}

The authors declare no conflict of interest.

\section{ACKNOWLEDGEMENTS}

We are grateful to Dr Mary-Claire King for innumerable discussions and suggestions. We also thank the members of Family $\mathrm{C}$ for cooperation in this study. This work was supported by the Scientific and Technological Research Council of Turkey (TUBITAK-SBAG 108S036 and 108S355) and Turkish Academy of Sciences (TUBA research support) to TO; Yale Program on Neurogenetics, the Yale Center for Human Genetics and Genomics and National Institutes of Health grants RC02 NS070477 to MG.

1 Tan U: A new syndrome with quadrupedal gait, primitive speech, and severe mental retardation as a live model for human evolution. Int J Neurosci 2006; 116 : 361-369.

2 Turkmen S, Demirhan O, Hoffmann $\mathrm{K}$ et al: Cerebellar hypoplasia and quadrupedal locomotion in humans as a recessive trait mapping to chromosome 17p. J Med Genet 2006; 43: 461-464.

3 Ozcelik T, Akarsu N, Uz E et al: Mutations in the very low-density lipoprotein receptor VLDLR cause cerebellar hypoplasia and quadrupedal locomotion in humans. Proc Nat/ Acad Sci USA 2008; 105: 4232-4236.

4 Moheb LA, Tzschach A, Garshasbi M et al: Identification of a nonsense mutation in the very low-density lipoprotein receptor gene (VLDLR) in an Iranian family with dysequilibrium syndrome. Eur J Hum Genet 2008; 16: 270-273.

5 Kolb LE, Arlier Z, Yalcinkaya C et al: Novel VLDLR microdeletion identified in two Turkish siblings with pachygyria and pontocerebellar atrophy. Neurogenetics 2010; 11: 319-325.

6 Turkmen S, Guo G, Garshasbi M et al: CA8 mutations cause a novel syndrome characterized by ataxia and mild mental retardation with predisposition to quadrupedal gait. PLoS Genet 2009; 5: e1000487.

7 Gulsuner S, Tekinay AB, Doerschner $\mathrm{K}$ et al: Homozygosity mapping and targeted genomic sequencing reveal the gene responsible for cerebellar hypoplasia and quadrupedal locomotion in a consanguineous kindred. Genome Res 2011; 21: 1995-2003.

8 Boycott KM, Flavelle S, Bureau A et al: Homozygous deletion of the very low density lipoprotein receptor gene causes autosomal recessive cerebellar hypoplasia with cerebral gyral simplification. Am J Hum Genet 2005; 77: 477-483.

9 Tan U: Evidence for 'Unertan Syndrome' and the evolution of the human mind. Int $J$ Neurosci 2006; 116: 763-774.
10 Cacciagli P, Haddad MR, Mignon-Ravix C et al: Disruption of the ATP8A2 gene in a patient with a $\mathrm{t}(10 ; 13)$ de novo balanced translocation and a severe neurological phenotype. Eur J Hum Genet 2010; 18: 1360-1363.

11 Seelow D, Schuelke M, Hildebrandt F et al: HomozygosityMapper-an interactive approach to homozygosity mapping. Nucleic Acids Res 2009; 37: W593-W599.

$12 \mathrm{Li} \mathrm{H}$, Ruan J, Durbin R: Mapping short DNA sequencing reads and calling variants using mapping quality scores. Genome Res 2008; 18: 1851-1858.

$13 \mathrm{Li} \mathrm{H}$, Handsaker B, Wysoker A et al: The Sequence Alignment/Map format and SAMtools. Bioinformatics 2009; 25: 2078-2079.

$14 \mathrm{Li} \mathrm{H}$, Durbin R: Fast and accurate long-read alignment with Burrows-Wheeler transform. Bioinformatics 2010; 26: 589-595.

15 Quinlan AR, Hall IM: BE DTools: a flexible suite of utilities for comparing genomic features. Bioinformatics 2010; 26: 841-842.

16 Flicek P, Amode MR, Barrell D et al: Ensembl 2011. Nucleic Acids Res 2011; 39. D800-D806.

$17 \mathrm{Ng}$ PC, Henikoff S: Predicting deleterious amino acid substitutions. Genome Res 2001; 11: 863-874.

18 Adzhubei IA, Schmidt S, Peshkin L et al: A method and server for predicting damaging missense mutations. Nat Methods 2010; 7: 248-249.

19 Schwarz JM, Rödelsperger C, Schuelke M et al: MutationTaster evaluates diseasecausing potential of sequence alterations. Nat Methods 2010; 7: 575-576.

20 Davydov EV, Goode DL, Sirota M et al: Identifying a high fraction of the human genome to be under selective constraint using GERP + +. PLoS Comput Biol 2010; 6: e1001025.

21 Cooper GM, Stone EA, Asimenos G et al: Distribution and intensity of constraint in mammalian genomic sequence. Genome Res, 2005; 15: 901-913.

22 Finn RD, Mistry J, Tate J et al: The Pfam protein families database. Nucleic Acids Res 2010; 38: D211-D222.

23 Bryson K, McGuffin LJ, Marsden RL et al: Protein structure prediction servers at University College London. Nucleic Acids Res 2005; 33: W36-W38.

24 Hartl D, Irmler M, Romer I et al: Transcriptome and proteome analysis of early embryonic mouse brain development. Proteomics 2008; 8: 1257-1265.

25 Huang da W, Sherman BT, Lempicki RA: Systematic and integrative analysis of large gene lists using DAVID bioinformatics resources. Nat Protoc 2009; 4: 44-57.

26 Rozen S, Skaletsky H: Primer3 on the WWW for general users and for biologist programmers. Methods Mol Biol 2000; 132: 365-386.

27 Pfaffl MW: A new mathematical model for relative quantification in real-time RT-PCR. Nucleic Acids Res 2001; 29: e45.

28 Halleck MS, Lawler JFJR, Blackshaw S et al: Differential expression of putative transbilayer amphipath transporters. Physiol Genomics 1999; 1: 139-150.

29 Graham TR, Kozlow MM: Interplay of proteins and lipids in generating membrane curvature. Curr Opin Cell Biol 2010; 22: 430-436.

30 Puts CF, Holthuis JC: Mechanism and significance of $\mathrm{P}_{4}$ ATPase-catalyzed lipid transport: Lessons from a Na+/K+-pump. Biochim Biophys Acta 2009; 1791: 603-611.

31 Meguro M, Kashiwagi A, Mitsuya K et al: A novel maternally expressed gene, ATP10C, encodes a putative aminophospholipid translocase associated with Angelman syndrome. Nat Genet 2001; 28: 19-20.

32 Stapelbroek JM, Peters TA, vanBeurden DH et al: ATP8B1 is essential for maintaining normal hearing. Proc Natl Acad Sci USA 2009; 106: 9709-9714.

33 Klomp LWJ, Vargas JC, van Mil SWC et al: Characterization of mutations in ATP8B1 associated with hereditary cholestasis. Hepatology 2004; 40: 27-38.

34 Coleman JA, Kwok MC, Molday RS: Localization, purification, and functional reconstitution of the P4-ATPase Atp8a2, a phosphatidylserine flippase in photoreceptor disc membranes. J Biol Chem 2009; 284: 32670-32679.

35 Wu C, Orozco C, Boyer J et al: BioGPS: an extensible and customizable portal for querying and organizing gene annotation resources. Genome Biol 2009; 10: R130.

36 Sarac 0 , Gulsuner S, Yildiz-Tasci Y et al: Neuro-opthalmologic findings in humans with quadrupedal locomotion. Ophthalmic Genet 2012; e-pub ahead of print 11 June 2012; PMID: 22686558.

37 Cohen D, Bar-Yosef U, Levy J et al: Homozygous CRYBB1 deletion mutation underlies autosomal recessive congenital cataract. Invest Ophthalmol Vis Sci 2007; 48: 2208-2213.

38 Harel T, Rabinowitz R, Hendler N et al: COL11A2 mutation associated with autosomal recessive Weissenbacher-Zweymuller syndrome: molecular and clinical overlap with otospondylomegaepiphyseal dysplasia (OSMED). Am J Med Genet A 2005; 132: 33-35.

39 Markus B, Narkis G, Landau D et al: Autosomal recessive lethal congenital contractural syndrome type 4 (LCCS4) caused by a mutation in MYBPC1. Hum Mutat 2012; e-pub ahead of print 18 May 2012; doi:10.1002/humu.22122; PMID: 22610851

Supplementary Information accompanies the paper on European Journal of Human Genetics website (http://www.nature.com/ejhg) 